\title{
Analytic Scattering and Refraction Models for Exoplanet Transit Spectra
}

\author{
Tyler D. Robinson ${ }^{1,3,4}$ (D) , Jonathan J. Fortney ${ }^{1,3}$ (1) , and William B. Hubbard ${ }^{2}$ (1) \\ ${ }^{1}$ Department of Physics and Astronomy, Northern Arizona University, Flagstaff, AZ 86011, USA; tyler.robinson@nau.edu \\ ${ }^{2}$ Lunar and Planetary Laboratory, University of Arizona, Tucson, AZ 85721-0092, USA \\ Received 2017 February 7; revised 2017 August 18; accepted 2017 October 18; published 2017 November 27
}

\begin{abstract}
Observations of exoplanet transit spectra are essential to understanding the physics and chemistry of distant worlds. The effects of opacity sources and many physical processes combine to set the shape of a transit spectrum. Two such key processes-refraction and cloud and/or haze forward-scattering-have seen substantial recent study. However, models of these processes are typically complex, which prevents their incorporation into observational analyses and standard transit spectrum tools. In this work, we develop analytic expressions that allow for the efficient parameterization of forward-scattering and refraction effects in transit spectra. We derive an effective slant optical depth that includes a correction for forward-scattered light, and present an analytic form of this correction. We validate our correction against a full-physics transit spectrum model that includes scattering, and we explore the extent to which the omission of forward-scattering effects may bias models. Also, we verify a common analytic expression for the location of a refractive boundary, which we express in terms of the maximum pressure probed in a transit spectrum. This expression is designed to be easily incorporated into existing tools, and we discuss how the detection of a refractive boundary could help indicate the background atmospheric composition by constraining the bulk refractivity of the atmosphere. Finally, we show that opacity from Rayleigh scattering and collision-induced absorption will outweigh the effects of refraction for Jupiter-like atmospheres whose equilibrium temperatures are above 400-500 K.
\end{abstract}

Key words: planets and satellites: atmospheres - radiative transfer - scattering

\section{Introduction}

Transit spectroscopy (Seager \& Sasselov 2000; Brown 2001; Hubbard et al. 2001) is an important technique used in the study of exoplanet atmospheric composition. Observations of transit spectra are now a common approach to studying a diversity of planet types with NASA's Hubble Space Telescope (Fraine et al. 2014; Knutson et al. 2014; Kreidberg et al. 2014; Sing et al. 2016). Exoplanet transit spectroscopy will also be a key science component of NASA's James Webb Space Telescope (JWST; Gardner et al. 2006), which is expected to provide high-quality transit spectra of many tens of targets over the duration of the mission (Beichman et al. 2014).

Recently, physical processes that were omitted from early models of exoplanet transit spectra have been shown to be of significant importance in certain circumstances. In particular, atmospheric refraction can lead to "floors" in transit spectra of worlds with relatively transparent atmospheres, or worlds that are located far from their host star so that the angular size of the host star as seen from the planet is small (Sidis \& Sari 2010; Muñoz et al. 2012; Bétrémieux \& Kaltenegger 2014, 2015; Misra et al. 2014; Dalba et al. 2015; Bétrémieux 2017). Additionally, forward-scattering from atmospheric clouds and hazes has been shown to be an important consideration for exoplanets whose host stars are of relatively large angular size as seen from the planet (De Kok \& Stam 2012; Robinson 2017).

Incorporating refraction and/or scattering into a transit spectrum model typically comes at great computational cost. For the former, ray-tracing simulations need to be implemented, and the latter warrants three-dimensional Monte Carlo techniques. Thus, typical transit tools, which place

\footnotetext{
${ }^{3}$ University of California, Santa Cruz, Other Worlds Laboratory.

${ }^{4}$ NASA Astrobiology Institute's Virtual Planetary Laboratory.
}

a strong emphasis on computational efficiency, neglect the physics of refraction or scattering (e.g., Barstow et al. 2012; Benneke \& Seager 2012; Howe \& Burrows 2012; Line et al. 2012; Lee et al. 2014; Waldmann et al. 2015; Morley et al. 2017).

Given the desire to balance realistic physics with computational efficiency in transit spectrum models, there is a clear need for the development of fast approaches to incorporating both refraction and scattering effects into standard modeling tools. Also, with the improvement in observational precision expected from JWST, now is an opportune time to examine the fundamentals of the physics that shape transit spectra. Here, we use simple physical arguments to derive an analytic correction that accounts for forward-scattering effects in transit, and we use previously published expressions to arrive at a simple equation for the pressure level of the refractive boundary for a transiting planet. These corrections are designed to be easily incorporated into any standard transit spectrum model, and can also be used to understand the basic physics of refraction and scattering in transit spectra. To ensure validity, we compare results from key expressions against those from more sophisticated techniques. Finally, we use the refractive boundary treatment to explain the circumstances under which refraction is likely to be an important process in shaping the transit spectra of worlds whose atmospheres are primarily $\mathrm{H}_{2}$ and $\mathrm{He}$.

\section{Theory}

In transit spectroscopy, the fundamental consideration is whether or not rays that traverse an exoplanet atmosphere during transit connect the observer to the stellar disk. A patch on the disk of a transiting exoplanet will appear opaque if rays emerging from this location are strongly attenuated by gas or 
aerosol absorption opacity, or if these rays experience enough refraction to bend them off the host stellar disk. Scattering opacity can also cause the patch to become opaque, since aerosols that are not strongly forward-scattering are likely to direct rays away from the host stellar disk. Considering all of these processes, it is apparent that the key quantities that determine the transmitted light emerging from a patch on the disk of a transiting exoplanet are: the slant absorption optical depth, $\tau_{\mathrm{a}}$, the slant-scattering optical depth, $\tau_{\mathrm{s}}$, the normalized scattering phase function, $P(\theta)$ (where $\theta$ is the scattering angle), the refraction angle, $\omega$, the projected separation between the patch and the opposite limb of the stellar host, and the angular size of the host star as seen from the planet. A number of these quantities depend on wavelength, and we leave this dependence implicit for cleaner presentation.

The following subsections present simple models that enable scattering and refraction effects to be easily incorporated into standard transit spectrum tools. We derive these using the typical assumption that the entire planetary disk overlays a star of uniform surface brightness, as limb-darkening is accounted for in standard transit observation data reduction procedures (e.g., Mandel \& Agol 2002; Kreidberg 2015). We also assume that the planet is centered on the stellar disk, as this is the most straightforward regime for computing transit spectra, although we note pieces of our theory that can be easily generalized for cases that break our adopted symmetry. Finally, our theory will not account for brightening effects related to refraction (Misra \& Meadows 2014) or forward-scattering (Robinson 2017; García Muñoz et al. 2017) while the planet is slightly off or partially overlapping the stellar disk.

\subsection{Scattering Effects}

Standard transit spectrum models treat all optical depth (i.e., the combined absorption and scattering optical depths) as absorption optical depth. However, the forward-scattered portion of a ray will still be directed toward the observer. Thus, an improved treatment of attenuation in transit spectra would reduce the scattering optical depth by an amount that depends on the extent of the forward-scattering peak in the scattering phase function, and then equate the remaining scattering and absorption optical depths with the effective absorption optical depth.

To begin, we adopt the $\delta$-isotropic approximation to radiative transfer (Thomas \& Stamnes 1999, their Section 6.8.1), where the scattering phase function is represented by an isotropic component and a forward component of strength $f$ (with $0 \leqslant f \leqslant 1$ ). Here, the radiant intensity, $I$, along a path (which we imagine as the slant path) would obey,

$$
\frac{d I}{d \tau_{\mathrm{e}}}=-I+f \tilde{\omega}_{0} I+\frac{\tilde{\omega}_{0}(1-f)}{4 \pi} \int_{4 \pi} I\left(\Omega^{\prime}\right) d \Omega^{\prime},
$$

where $\tau_{\mathrm{e}}$ is the extinction optical depth (i.e., the sum of the absorption and scattering optical depths), $\tilde{\omega}_{0}$ is the single scattering albedo, and $\Omega$ represents a solid angle. The final term in Equation (1) comes from the isotropic component of the phase function, which has been shown to be negligible for transit spectra (Brown 2001; Hubbard et al. 2001; Robinson 2017), so we choose to omit this term. With these assumptions, the radiative transfer equation can be written simply as

$$
\frac{d I}{d \tau_{\mathrm{eff}}}=-I
$$

where we have defined an effective optical depth that includes a correction for forward-scattering as

$$
d \tau_{\text {eff }}=\left(1-f \tilde{\omega}_{0}\right) d \tau_{\mathrm{e}} .
$$

It is widely known in the radiative transfer literature (e.g., Petty 2006, their Appendix A.2) that there is no unique way to set the value of the forward-scattering correction factor. Given the geometry of the problem at hand, in which we imagine back-tracing rays from an observer into the planetary atmosphere, where these rays then scatter into a cone of radiation that either fully or partially overlaps the host stellar disk, we suggest that the corrective factor should be based on the portion of the scattering phase function that is within the angular size of the stellar disk. This would then give,

$$
f=2 \pi \int_{0}^{\frac{R_{\mathrm{S}}}{a}} P(\theta) \sin (\theta) d \theta=2 \pi \int_{1-\frac{R_{\mathrm{S}}}{a}}^{1} P(\mu) d \mu,
$$

where $R_{\mathrm{s}}$ is the stellar radius, $a$ is the planet-star physical separation, $\mu=\cos (\theta)$, and we have assumed that $\sin ^{-1}\left(R_{\mathrm{S}} / a\right) \approx R_{\mathrm{s}} / a$. (Alternatively, see Budaj et al. 2015, their Equation (19), or DeVore et al. 2016, their Equation (A13).)

In practice, Equation (3) would simply be used in place of the extinction optical depth in any standard transit spectrum model. Such models typically operate by integrating the optical depth along a slant path, which yields a transmissivity through the atmosphere for a given impact parameter. In such a setup, a layer along the slant path that has any scattering optical depth (i.e., $\tilde{\omega}_{0}>0$ ) would have the slant extinction optical depth across the layer reduced by $1-f \tilde{\omega}_{0}$. Thus, our proposed correction is computationally inexpensive to implement inside of existing tools.

In the most general case, the integral in Equation (3) would need to be computed for each layer given a realistic scattering phase function. This computational burden is reduced if the scattering phase function is expanded in terms of Legendre polynomials. However, given that our correction factor is approximate, such sophistications are unwarranted. We propose an analytic correction factor that is based on the widely used Henyey-Greenstein phase function (Henyey \& Greenstein 1941), with

$$
P(\theta)=\frac{1}{4 \pi} \frac{1-g^{2}}{\left[1+g^{2}-2 g \cos (\theta)\right]^{3 / 2}},
$$

where $g$ is the asymmetry parameter. The correction factor for this phase function is then,

$$
\begin{aligned}
& f_{\mathrm{HG}}\left(g, R_{\mathrm{s}} / a\right)=\frac{1-g^{2}}{2 g} \\
& \quad \times\left[\frac{1}{1-g}-\frac{1}{\sqrt{1+g^{2}-2 g \cos \left(\frac{R_{\mathrm{s}}}{a}\right)}}\right] .
\end{aligned}
$$

Additional complexity could be added with little computational cost by using a two-term Henyey-Greenstein phase function 


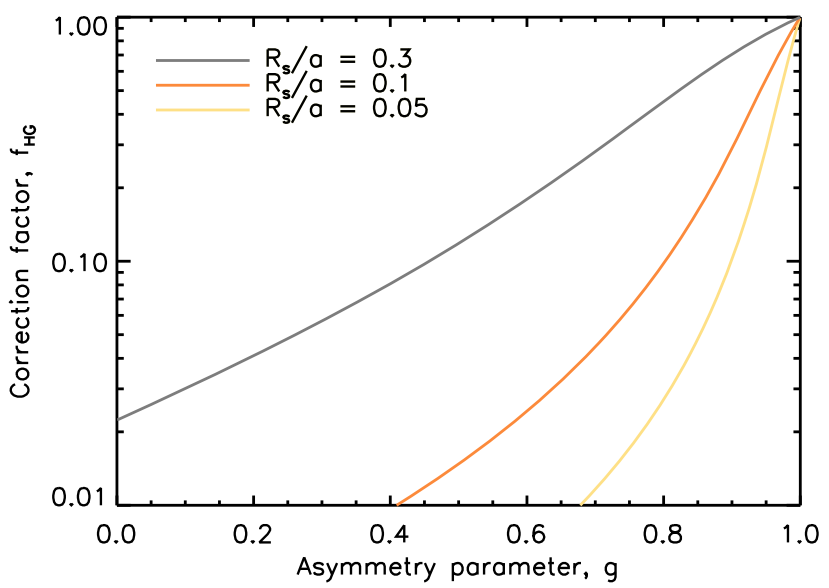

Figure 1. Forward-scattering correction factor, assuming a Henyey-Greenstein phase function, as a function of asymmetry parameter, $g$, for several different host star angular sizes.

(Irvine 1965), whose correction factor would be,

$$
\begin{aligned}
f_{\mathrm{TTHG}}\left(g_{1}, g_{2}, w, R_{\mathrm{s}} / a\right)= & w \cdot f_{\mathrm{HG}}\left(g_{1}, R_{\mathrm{s}} / a\right) \\
& +(1-w) \cdot f_{\mathrm{HG}}\left(g_{2}, R_{\mathrm{s}} / a\right),
\end{aligned}
$$

where $g_{2}<0$ causes an enhancement in backward-scattering, and $w$ (whose value is between zero and unity) is a weighting factor for the forward- versus backward-scattering terms. Figure 1 demonstrates the scale of the correction factor from Equation (6) as a function of the asymmetry parameter and for several different host star angular sizes. When adopting the correction appropriate for the Henyey-Greenstein phase function, the correction factor only varies as rapidly as does the asymmetry parameter.

Briefly, we note that, for an atmosphere where the dominant opacity source is an aerosol that is distributed vertically with some scale height, $H_{\mathrm{h}}$ (where the sub-script "h" is for "haze"), we can use Equation (8) from Robinson et al. (2014) to derive a radius (or altitude) difference probed when our correction is included versus omitted. The transit radius, $r$, would be set where the slant optical depth is of order unity, and would satisfy,

$$
1 \approx \tau_{0} \sqrt{\frac{2 \pi r}{H_{\mathrm{h}}}} e^{-\left(r-R_{\mathrm{p}}\right) / H_{\mathrm{h}}},
$$

where $\tau_{0}$ is a reference vertical extinction optical depth, $R_{\mathrm{p}}$ is the planetary radius, and we have omitted the wavelength dependence from the original expression. Replacing $\tau_{0}$ with an optical depth that includes our forward-scattering correction (i.e., $\left[1-f \tilde{\omega}_{0}\right] \tau_{0}$ ) would produce a similar expression for the transit radius probed when forward-scattering is considered, which we will call $r^{\prime}$. Taking the ratio of these two expressions and solving for the radius difference relative to the haze scale height gives

$$
\frac{r-r^{\prime}}{H_{\mathrm{h}}}=\frac{\Delta r}{H_{\mathrm{h}}}=-\ln \left(1-f \tilde{\omega}_{0}\right),
$$

where we have assumed that $\Delta r \ll r$. For these same angular sizes of the host star as in Figure 1, and for the adopted Henyey-Greenstein phase function, Figure 2 demonstrates Equation (9), assuming pure scattering and with $\tilde{\omega}_{0}=1$. The

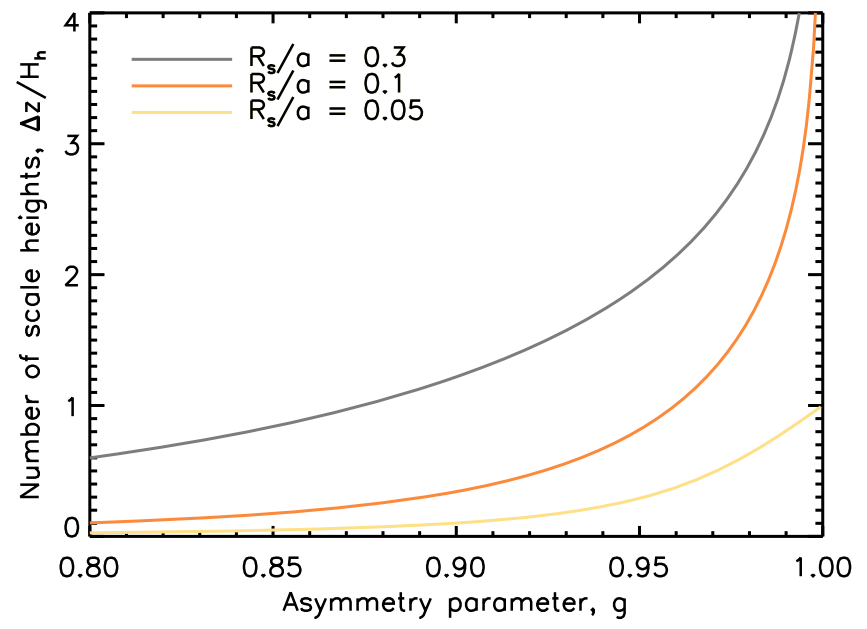

Figure 2. Difference in the altitude probed when our forward-scattering correction is included vs. excluded, plotted relative to the haze scale height and taken from Equation (9). We assume a purely scattering haze, and adopt a Henyey-Greenstein phase function as in Figure 1. Curves are for different host star angular sizes.

range of scale height biases shown in Figure 2 (i.e., $\lesssim 4$ ) agrees with quoted results from the Monte Carlo scattering model of De Kok \& Stam (2012).

\subsection{Refraction Effects}

Following Hubbard et al. (1988), we derive the total refractive bending angle by determining the phase change, $\Phi$, of a wave passing on a straight-line path through the exoplanet atmosphere. Since the refractivity, $\nu$, of the atmosphere is proportional to the number density, and assuming these to both be distributed exponentially in an isothermal atmosphere with scale height $H$, we have,

$$
\Phi=k \nu_{0} H \sqrt{\frac{2 \pi r}{H}} e^{-\left(b-r_{0}\right) / H},
$$

where $k$ is the wavenumber, $\nu_{0}$ is the refractivity evaluated at a radius $r_{0}$ in the planetary atmosphere, and $r$ is the radius of closest approach for the ray (which is equivalent to the impact parameter for our assumed straight-line path). The total refractive bending angle (taken to be non-negative) is then

$$
\omega=-\frac{1}{k} \frac{\partial \Phi}{\partial r}=-\nu_{0}\left(\frac{1}{2} \sqrt{\frac{2 \pi H}{r}}-\sqrt{\frac{2 \pi r}{H}}\right) e^{-\left(r-r_{0}\right) / H} .
$$

Since $r \gg H$, we have

$$
\omega \approx \nu_{0} \sqrt{\frac{2 \pi r}{H}} e^{-\left(r-r_{0}\right) / H},
$$

which is an expression first derived by Fabry (1929, their Equation (4)), and was also derived by Baum \& Code (1953, their Equation (6)).

Sidis \& Sari (2010) noted that the base of the transit spectrum (i.e., the smallest planetary radius probed, $r_{\min }$ ) would be determined by Equation (12) when the refraction effect is large enough to cause a ray coming from one side of the planet to bend off the far limb of the star, which would give

$$
\frac{R_{\mathrm{s}}+R_{\mathrm{p}}}{a} \approx \nu_{0} \sqrt{\frac{2 \pi r_{\min }}{H}} e^{-\left(r_{\min }-r_{0}\right) / H} .
$$


Table 1

Gas Refractivity Expressions at STP

\begin{tabular}{|c|c|c|c|}
\hline Gas & Refractivity, $\nu$ (with $\sigma=1 \mu \mathrm{m} / \lambda$ ) & References & Note(s) \\
\hline$\overline{\mathrm{H}_{2}}$ & $1.48956 \times 10^{-2} /\left(180.7-\sigma^{2}\right)+4.9037 \times 10^{-3} /\left(92-\sigma^{2}\right)$ & Peck \& Huang (1977) & $0.2 \mu \mathrm{m}<\lambda<1.7 \mu \mathrm{m}$ \\
\hline $\mathrm{He}$ & $\begin{array}{l}0.01470091 /\left(423.98-\sigma^{2}\right) \\
6.991 \times 10^{-2} /\left(166.175-\sigma^{2}\right) \\
+1.4472 \times 10^{-3} /\left(79.609-\sigma^{2}\right)\end{array}$ & Mansfield \& Peck (1969) & $0.5 \mu \mathrm{m}<\lambda<2.1 \mu \mathrm{m}$ \\
\hline $\mathrm{CO}_{2}$ & $\begin{array}{l}+6.42941 \times 10^{-5} /\left(56.3064-\sigma^{2}\right) \\
+5.21306 \times 10^{-5} /\left(46.0196-\sigma^{2}\right) \\
+1.46847 \times 10^{-6} /\left(0.0584738-\sigma^{2}\right)\end{array}$ & Bideau-Mehu et al. (1973) & $0.2 \mu \mathrm{m}<\lambda<2.5 \mu \mathrm{m}$ \\
\hline $\mathrm{N}_{2}$ & $6.8552 \times 10^{-5}+3.243157 \times 10^{-2} /\left(144-\sigma^{2}\right)$ & Peck \& Nath Khanna (1966) & $0.2 \mu \mathrm{m}<\lambda<2.5 \mu \mathrm{m}$ \\
\hline $\mathrm{O}_{2}$ & $\begin{array}{l}1.26805 \times 10^{-4}+1.04202 \times 10^{-2} /\left(75.4-\sigma^{2}\right) \\
3.011 \times 10^{-2} /\left(124.40-\sigma^{2}\right)\end{array}$ & Křen (2011) & $0.4 \mu \mathrm{m}<\lambda<1.8 \mu \mathrm{m}$ \\
\hline $\mathrm{H}_{2} \mathrm{O}$ & $\begin{array}{l}+7.46 \times 10^{-3} \cdot(0.203-\sigma) \\
\left(1.03-1.98 \times 10^{3} \sigma^{2}+8.1 \times 10^{4} \sigma^{4}-1.7 \times 10^{8} \sigma^{8}\right)^{-1}\end{array}$ & Hill \& Lawrence (1986) & $0.36 \mu \mathrm{m}<\lambda<19 \mu \mathrm{m}$ \\
\hline
\end{tabular}

Note.

${ }^{\mathrm{a}}$ See also Polyanskiy (2016).

Evaluating this expression yields the minimum impact parameter (or radius, equivalently) probed (Sidis \& Sari 2010, their Equation (17)). Bétrémieux \& Kaltenegger (2014), who called the bending angle highlighted by Sidis \& Sari (2010) the "critical deflection" angle, expressed Equation (13) in terms of the maximum atmospheric number density probed (in amagats; their Equation (11)).

Instead of expressing the maximum depth probed in terms of number density or radius, we propose that the more intuitive measure is atmospheric pressure, $p$. Taking $p=p_{0} \exp \left[-\left(r-r_{0}\right) / H\right]$, where $p_{0}$ is the reference pressure at $r_{0}$, we can use the result of Sidis \& Sari (2010) to determine the maximum pressure probed by the ray, $p_{\max }$, as,

$$
p_{\max }=p_{0} \frac{1}{\nu_{0}} \frac{R_{\mathrm{s}}+R_{\mathrm{p}}}{a} \sqrt{\frac{H}{2 \pi r_{0}}} \approx p_{0} \frac{1}{\nu_{0}} \frac{R_{\mathrm{s}}}{a} \sqrt{\frac{H}{2 \pi r_{0}}},
$$

where the approximation assumes $R_{\mathrm{p}} \ll R_{\mathrm{s}}$. For easy use, we can adopt Jupiter-like values and write,

$$
\begin{aligned}
p_{\max }= & 23 \mathrm{mbar} \cdot\left(\frac{1.23 \times 10^{-4}(\mathrm{STP})}{\nu_{0}}\right)\left(\frac{T}{130 \mathrm{~K}}\right)^{3 / 2}\left(\frac{R_{\mathrm{s}}}{R_{\odot}}\right) \\
& \times\left(\frac{5.2 \mathrm{au}}{a}\right)\left(\frac{R_{\mathrm{J}}}{R_{\mathrm{p}}}\right)^{1 / 2}\left(\frac{2.2 \mathrm{amu}}{\mu}\right)^{1 / 2}\left(\frac{24.8 \mathrm{~m} \mathrm{~s}^{-2}}{g}\right)^{1 / 2},
\end{aligned}
$$

where $R_{\mathrm{p}}$ is taken as the 1 bar planetary radius, $\mu$ is the mean molar weight for the atmosphere, $g$ is the acceleration due to gravity, and the refractivity is given at standard temperature and pressure (STP; $273 \mathrm{~K}$ and $1.013 \mathrm{bar}$ ). Note that including the planetary radius on the right side of Equation (14) serves to increase the maximum pressure probed by a factor of $R_{\mathrm{p}} / R_{\mathrm{s}}$, and this omission is unlikely to be a limiting factor (as compared to, e.g., the isothermal assumption). Omitting the planetary radius contribution is justified, as exoplanet transit depths tend to be smaller than 2\%-3\% (implying $R_{\mathrm{p}} / R_{\mathrm{s}}$ less than 0.2$),{ }^{5}$ and furthermore, radial velocity results indicate that massive planets are relatively rare around low-mass stars (Johnson et al. 2010). Table 1 contains expressions for

\footnotetext{
See: exoplanetarchive.ipac.caltech.edu.
}

computing the wavelength-dependent refractivity at STP for a number of key gases.

In Equation (14), we note that the only term that depends on wavelength is the refractivity (which tends to vary weakly with wavelength across the near-infrared and thermal infrared). Also, for cases that aim to compute transit spectra that break our assumed symmetry where the planet centered on the stellar disk, Equation (13) would need to be evaluated for a grid of locations around the planetary disk. Each angular point would have a different refractive boundary pressure, and an areaweighted average could be performed for the grid (in angle) of transit spectra for the planet.

\section{Results}

The scattering correction model and the refraction expression given above are easily implemented in transit spectrum tools. Here, we verify these simple expressions via comparisons to previously published analyses and to more sophisticated models. We conclude this section using the refraction model to understand the conditions under which refractive bending of light is likely to be important for atmospheres of Jupiter-like planets.

\subsection{Model Verifications}

We verify our scattering correction by first reproducing a numerical experiment from Robinson (2017, their Section 4.2). Here, the transit depth of a narrow, cloud-filled annulus on the planetary disk was computed for a range of cloud slantscattering optical depths, scattering asymmetry parameters, and host star angular sizes. Beyond the cloud, no other opacity source was included, and the cloud was taken to be pure scattering with a single scattering phase function described by the Henyey-Greenstein phase function. Results were referenced to the "pure absorption" scenario, where all scattering optical depth is treated as absorption optical depth. Figure 3 shows the Monte Carlo cases in the left column and the cases where the analytic correction is used in the right column. The transit depth in the analytic case simply scales as $1-\operatorname{EXP}\left[(1-f) \Delta \tau_{\mathrm{s}}\right]$, where $\Delta \tau_{\mathrm{s}}$ is the slant-scattering optical depth of the cloud. Clearly, the analytic model reproduces the shape and scale of the results from the full Monte Carlo simulation. 

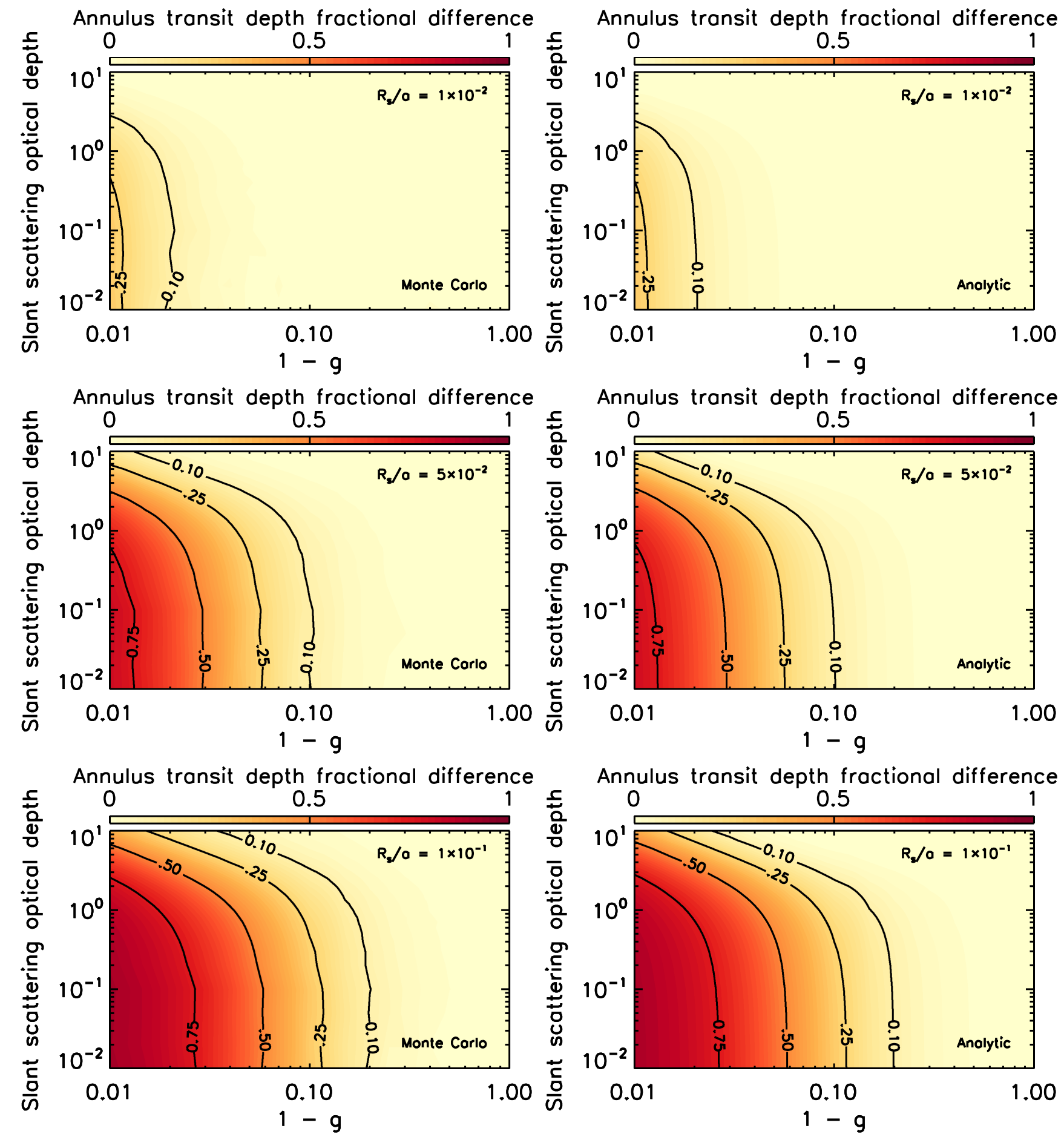

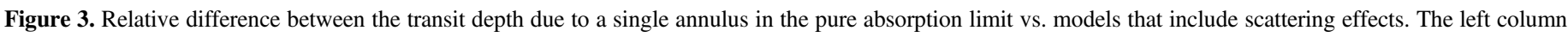

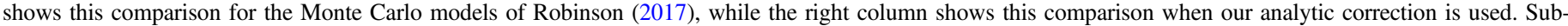

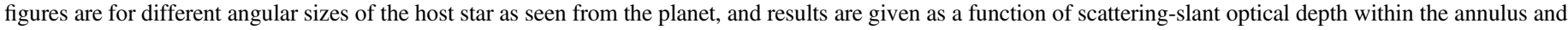
the scattering asymmetry parameter.

Next, we verify our scattering correction by comparing to full transit spectra computed according to the full-physics Monte Carlo model of Robinson (2017). For this comparison, we use a standardized hot Jupiter-like atmospheric model that has a planetary radius (at the 10 bar pressure level) of $1.16 R_{\mathrm{J}}$, a planetary mass of $1.14 M_{\mathrm{J}}$, a stellar radius of $0.78 R_{\odot}$, and atmospheric volume mixing ratios of $\mathrm{H}_{2}, \mathrm{He}$, and $\mathrm{H}_{2} \mathrm{O}$ of 0.85 , 0.15 , and $4 \times 10^{-4}$, respectively. The 126 model layers are placed evenly in log-pressure between 10 bar and $10^{-9}$ bar. The atmosphere is isothermal at $1500 \mathrm{~K}$ and the planet is located $0.031 \mathrm{au}$ from its host. Non-absorbing, forward-scattering ( $g=0.95)$ clouds of different slant-scattering optical depths are distributed across a pressure scale height, and are placed at different atmospheric pressure levels. Figure 4 compares transit spectra that use our proposed correction to models in the pure absorption limit, as well as to full-physics multiple-scattering models. Briefly, transit spectra using our correction were computed using Equations (5), (7), and (36) from Robinson 


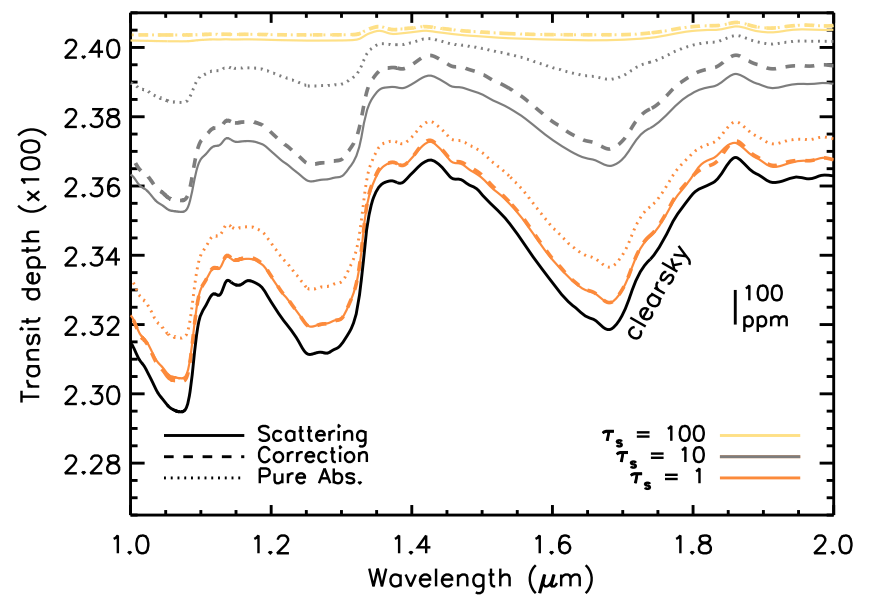

Figure 4. Transit spectra from the fully multiple-scattering model of Robinson (2017, solid), a model in the pure absorption limit (dotted) where all optical depth is treated as absorption optical depth, and from a model that adopts the correction factor in Equation (6) (dashed). Isolated, conservatively scattering clouds with different slant-scattering optical depths (indicated by color) are distributed across one pressure scale height centered at $10^{-4}$ bar in a hot Jupiter-like atmosphere. Additional model details are given in the text. An asymmetry parameter of $g=0.95$ is adopted. Features are due to water vapor, and a $100 \mathrm{ppm}$ bar is given for scale.

(2017), where the differential optical depth in these expressions was replaced with our effective optical depth. Clearly, the model that adopts our correction performs much better than the commonly used pure absorption model. Differences between the former and the multiple-scattering model are typically less than 30-50 ppm, while the latter typically has errors larger than 100-200 ppm (for the two thinnest cloud cases).

While expressions similar to Equation (14) have previously appeared in the literature (Sidis \& Sari 2010; Bétrémieux \& Kaltenegger 2014), little work has been done to explore the validity of this treatment. We verify Equation (14) (including the planetary radius term on the right side) by comparing to the numerically derived values from Bétrémieux (2017), who investigated the location of refractive boundaries in transiting Jupiter-like exoplanets. Following this work, we adopt $p_{0}$ to be 1 bar, $\nu_{0}$ to have a Jupiter-like value of $1.23 \times 10^{-4}$ at STP, and $r_{0}$ to be equal to Jupiter's radius of $6.99 \times 10^{4} \mathrm{~km}$. Additionally, like Bétrémieux (2017, their Equation (2)), we set the atmospheric temperature, $T$, to be equal to the equilibrium temperature for a world with Bond albedo, $A_{\mathrm{B}}$, and negligible internal heat, so that,

$$
\frac{a}{R_{\mathrm{s}}}=\frac{\sqrt{1-A_{\mathrm{B}}}}{2}\left(\frac{T_{\mathrm{s}}}{T}\right)^{2},
$$

where $T_{\mathrm{s}}$ is the host stellar effective temperature, and the Bond albedo is taken to be 0.3 . Table 3 compares the maximum pressures probed from Equation (14) to the numerically derived values from Bétrémieux (2017) for the planetary temperatures and host star temperatures used in that study. Here, we see that the analytic expression reproduces the ray-tracing results to within $20 \%$ when $p_{\max }$ is below 10 bar, growing to many tens of percent when $p_{\max }$ is above 10 bar. For the cases with the larger values of $p_{\max }$, the angular size of the host star $\left(R_{\mathrm{S}} / a\right)$, and thus the refraction angle, is greater than about 0.03 , so our assumption that rays pass straight through the atmosphere breaks down-these rays are instead bent more significantly toward lower pressures and probe deeper regions of the atmosphere than our simple theory would indicate. However, this regime is unlikely to be important because molecular or cloud opacity will likely prevent rays from probing this deep into an atmosphere.

We can further test the simple treatment of refraction against a ray-tracing model described in Robinson (2017). For this test, we consider a planet with Earth's radius and mass orbiting at 1 au from a Sun-like star. We adopt an isothermal atmosphere at $255 \mathrm{~K}$ (i.e., Earth's equilibrium temperature), and an atmospheric composition of $\mathrm{N}_{2}$ and $\mathrm{H}_{2} \mathrm{O}$ with volume mixing ratios of 0.99 and 0.01 , respectively. According to Equation (14), this world would have a $p_{\max }$ of 0.18 bar at $1 \mu \mathrm{m}$, which occurs at an altitude of $12 \mathrm{~km}$. This value is in good agreement with results from García Muñoz et al. (2012), who found a refractive floor in Earth's transit spectrum at $11.6 \mathrm{~km}$ using analytic arguments and $13.2 \mathrm{~km}$ using numerical models. The analytic result from these authors assumed an isothermal atmosphere with a pressure scale height of $8 \mathrm{~km}$, which is nearly identical to the scale height in our isothermal case. Using numerical models, Misra et al. (2014) found a $p_{\max }$ of 0.3 bar for a realistic Earth/Sun pair and Bétrémieux \& Kaltenegger (2014) found a $p_{\max }$ of 0.17 bar. Differences among these predicted values for the location of a refractive boundary are likely due to the treatment of atmospheric temperature (i.e., isothermal for analytic cases versus varying with altitude in the numerical cases), and as mentioned in Robinson (2017), the integration lengths used within numerical ray-tracing schemes.

When computing a transit spectrum by performing an integral/sum over a grid of impact parameters (e.g., Bétrémieux \& Kaltenegger 2013, their Equation (4)), one simply sets the transmission to zero for all rays with impact parameters below this refractive boundary. Figure 5 shows transit spectra for our adopted Earth-sized planet produced by a fully realistic model with refraction, a model without refraction, and a non-refracting model that sets slant transmission to zero below the refractive boundary at $12 \mathrm{~km}$ above the surface. (Note that the effective transit altitude, $z_{\text {eff }}$, is defined by setting the wavelengthdependent transit depth equal to $\left[\left(R_{\mathrm{p}}+z_{\text {eff }}\right) / R_{\mathrm{s}}\right]^{2}$.) The model with the analytic refractive boundary improves quite significantly over a model that does not include refraction.

Expanding our calculation to cooler spectral types, we find that the refractive boundaries for dwarf K5, M0, and M5 stars occur at 32 mbar $(9 \mathrm{~km}), 41 \mathrm{mbar}(7 \mathrm{~km})$, and 78 mbar $(2 \mathrm{~km})$, respectively, where we place the planet at Earth equivalent insolation distances $(0.37,0.27$, and $0.047 \mathrm{au}$, respectively). These values are in general agreement with the ray-tracing results of Bétrémieux \& Kaltenegger (2014) for Earths around host stars of different spectral types, and small differences (less than $2 \mathrm{~km}$ ) can likely be attributed to these authors using a realistic Earth temperature-pressure profile. Figure 5 shows transit spectra of our Earth-sized planet for the K5 and M5 dwarf cases. For all cases, our approach of setting the transmission to zero for rays that probe deeper than $p_{\max }$ reproduces the ray-tracing model quite well.

\subsection{Refractive Boundaries in Jovian Atmospheres with Collision-induced Absorption (CIA)}

An open question in exoplanet transit spectroscopy and spectral retrieval is the degree to which refraction may influence transit observations of worlds with hydrogen-rich 

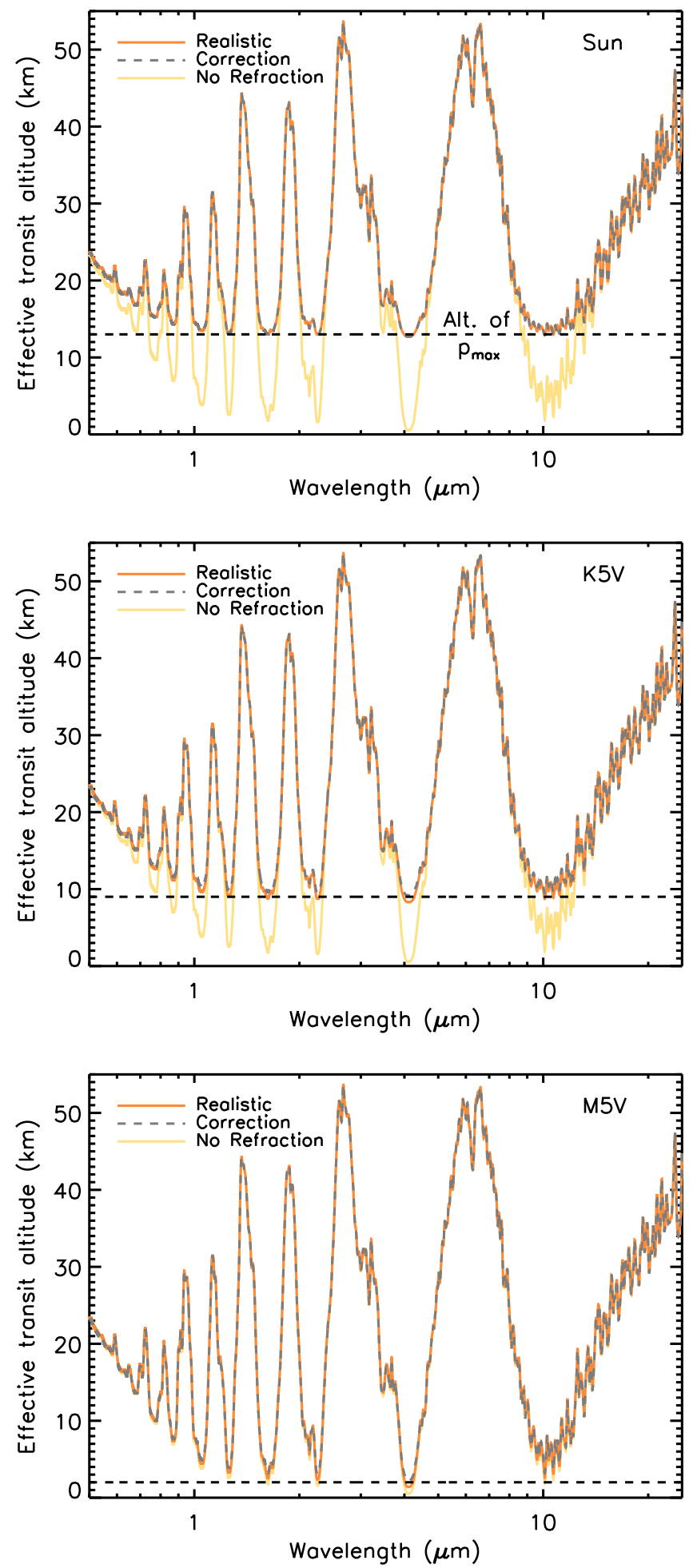

Figure 5. Transit spectra of an Earth-sized exoplanet at the Earth equivalent insolation distance for a Sun-like (top), K5 dwarf (middle), and M5 dwarf (bottom) host. Model atmosphere details are provided in the text. For each case, the refractive boundary from Equation (14) is indicated by a dashed black line. The colors indicate spectra from a ray-tracing model (orange), a model without refraction (yellow), and a model that includes a simple correction for refraction (gray) where the transmission is set to zero for rays that probe deeper than $p_{\max }$.

atmospheres. Refraction can be of first-order importance for worlds with nitrogen-rich atmospheres (Bétrémieux \& Kaltenegger 2014; Misra et al. 2014), but this is due to molecular nitrogen's lack of broad absorption bands or CIA features (except near $4.5 \mu \mathrm{m}$ Schwieterman et al. 2015). Molecular hydrogen has a broad CIA continuum that will compete with refraction (as well as clouds and Rayleigh scattering, Sidis \& Sari 2010) to set the limiting pressures probed between molecular absorption bands in transit spectrum observations. Here, we extend the study of Bétrémieux (2017), who investigated refraction effects in gas giant atmospheres but omitted both $\mathrm{H}_{2}-\mathrm{H}_{2}$ and $\mathrm{H}_{2}-\mathrm{He}$ CIA.

Figure 6 shows the pressure where the slant optical depth equals 0.56 for Rayleigh scattering and CIA for Jupiter-like worlds with isothermal atmospheres at 150, 300, and $500 \mathrm{~K}$. We assume a radius of $6.99 \times 10^{4} \mathrm{~km}$, a surface gravity of $24.8 \mathrm{~m} \mathrm{~s}^{-2}$, and a $0.9 / 0.1$ mixture of $\mathrm{H}_{2} / \mathrm{He}$ (by number). Rayleigh scattering and CIA from both hydrogen and helium (Abel et al. 2011) are included. Also shown are the pressures of the refractive boundary for host stars of different effective temperatures, from the non-approximate Equation (14). The angular size of the host star is determined using the energy balance expression in Equation (16) for an assumed Bond albedo of 0.3 . The pressure of the refractive boundary will depend weakly on Bond albedo, scaling as $\left(1-A_{\mathrm{B}}\right)^{-1 / 2}$.

In Figure 6, wavelengths where the refractive boundary curves sit above the Rayleigh or CIA curves are regions where refraction has the potential to limit observations (in the absence of any molecular absorption features from trace atmospheric species). The highest equilibrium temperatures where refraction will have a significant effect are roughly 400-500 K, depending on wavelength. For higher temperatures, the host star (as seen from the planet) has a relatively large angular size, thereby pushing the refractive boundary to pressures deeper than about 1-3 bar.

\section{Discussion}

The expressions given above are designed to be easily incorporated into existing transit spectrum models with little additional computational expense. For aerosol forward-scattering, one only need evaluate the correction factor (e.g., Equation (6)) for each layer while summing optical depths along a slant path. As shown in Figure 4, this correction makes a clear improvement over the commonly used pure absorption models. As outlined in Section 2.1, our forward-scattering correction can be used in cases where the angular symmetry about the planet disk is broken, and would result in more muted effects from asymmetric forwardscattering clouds on exoplanet terminators than are currently assumed in simulations (Line \& Parmentier 2016; MacDonald \& Madhusudhan 2017).

Our analysis, as shown in Figure 2, indicates that omitting a forward-scattering correction can cause a transit spectrum to be biased toward higher altitudes by as many as four aerosol scale heights. In other words, the effective transit altitude could be too large by several aerosol scale heights. Of course, this biasing depends on the host star angular size and the scattering phase function, among other parameters. As transit spectra typically probe several pressure scale heights, Figure 2 shows that forward-scattering effects can become comparable to molecular features when the aerosol scale height is comparable to (or larger than) the pressure scale height, when $g \gtrsim 0.85$, and when $R_{\mathrm{s}} / a \gtrsim 0.1$.

Regarding refraction, Equation (14) (see also Sidis \& Sari 2010, their Equation (17), and Bétrémieux \& Kaltenegger 2014, their Equation (11)) allows modelers and observers to quickly evaluate whether or not a refractive boundary is 

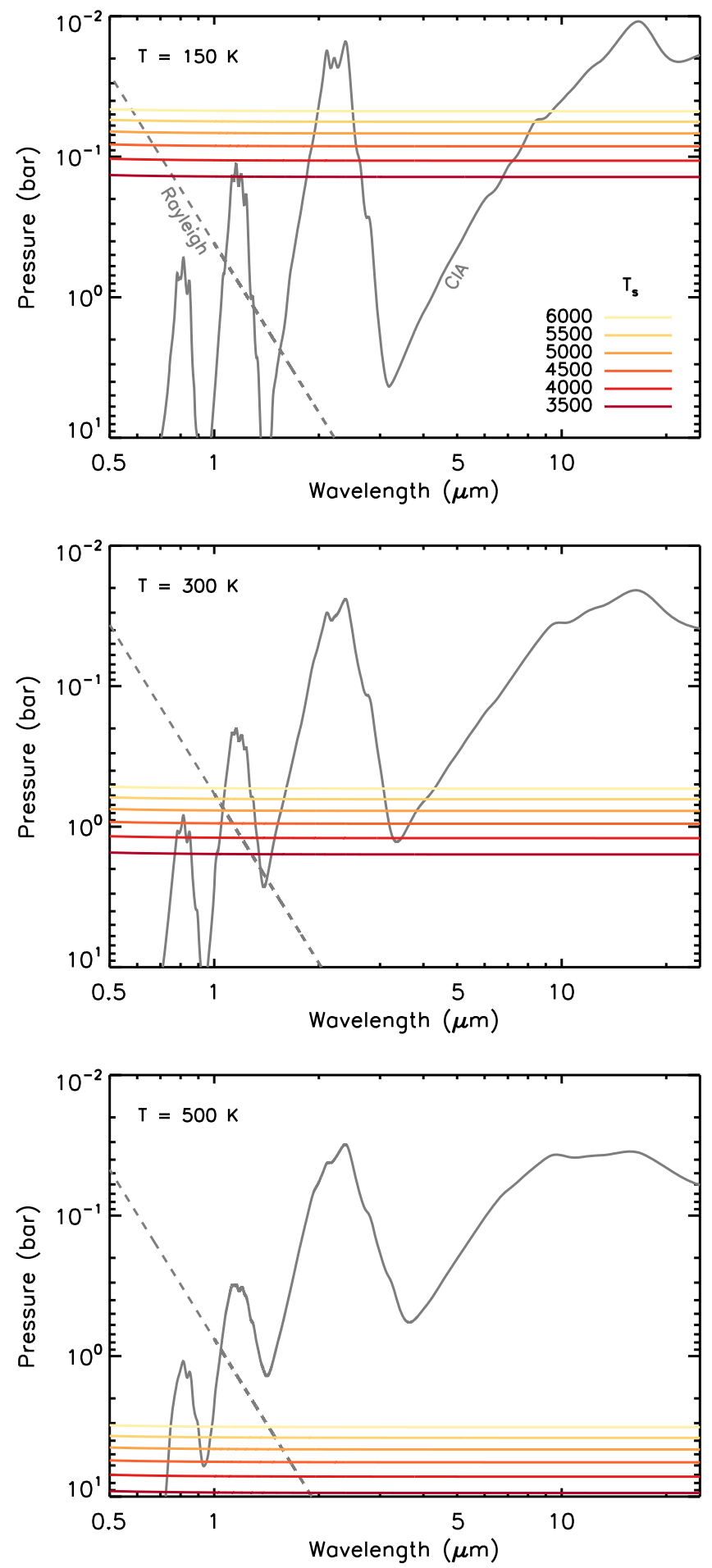

Figure 6. Pressure of slant optical depth equal to 0.5 for Rayleigh scattering (gray dashed) and CIA (gray solid) for Jovian worlds of different temperatures. The colored lines indicate the pressure of the refractive boundary (from Equation (14)) for host stars of different effective temperatures, where the angular size of the star is set by the energy balance required to yield the planetary temperature (Equation (16)). When horizontal colored lines fall below the gray curves, the refractive boundary is located at a higher pressure (or smaller altitude/radius) than where the transit spectrum would become opaque to Rayleigh scattering or CIA opacity, implying that refraction would not influence the shape of the transit spectrum.

likely to influence a given transit spectrum. Comparisons to previously published results from a ray-tracing model applied to gas giant exoplanets (Bétrémieux 2017) indicate that the expression is valid to within $10 \%-20 \%$ for the angular size of the host star $\left(R_{\mathrm{S}} / a\right)$ below about 0.03 (which correspond to maximum pressures below roughly 10 bar; see Table 2). At larger pressures, our assumption of a straight-line path for our rays breaks down, as rays would actually be bent to probe deeper into the exoplanet atmosphere. However, gas and aerosol extinction are likely to prevent observations from reaching such large pressures - the typical pressures probed in transit spectra are fractions of a bar.

In practice, if Equation (14) indicates that refraction may be an important consideration for a certain model or observation, then the effects of a refractive boundary can be mimicked by simply setting the slant transmission to zero below the refractive boundary when integrating over concentric annuli to produce a transit spectrum. Figure 5 demonstrates the efficacy of this simple approach across a wide range of angular sizes of the stellar host for Earth-sized planets with cloud-free, 1 bar $\mathrm{N}_{2}-\mathrm{H}_{2} \mathrm{O}$ atmospheres.

For retrieval studies, our forward-scattering correction factor shows that any cloud optical depth retrieved from an exoplanet transit observation is actually an "effective" optical depth that folds together information about the extent of the forwardscattering peak in the scattering phase function and the angular size of the host star. Also, Equation (14) indicates that, should a refractive boundary be detected in an exoplanet transit spectrum, inverse analyses may be able to use the location of this boundary to help constrain background atmospheric constituents, as this expression depends on the bulk atmospheric refractivity. However, the transit depth down to this refractive boundary depends on both the atmospheric scale height and the bulk refractivity (Sidis \& Sari 2010). Independently measuring the atmospheric scale height (through, e.g., detecting a Rayleigh scattering slope (Lecavelier Des Etangs et al. 2008)) would break this degeneracy. This characteristic of refractive boundaries is in addition to their ability to help break degeneracies when attempting to constrain abundances of trace atmospheric constituents (Bétrémieux 2017; Bétrémieux \& Swain 2016).

Finally, our analysis of the competing effects of refractive boundaries and Rayleigh scattering and CIA for Jupiter-like exoplanets provides a clear indication of the regimes where refraction will outweigh opacity from $\mathrm{H}_{2}$ and He. As shown in Figure 6, refraction can outweigh Rayleigh scattering and CIA for worlds whose equilibrium temperatures are below about $400-500 \mathrm{~K}$. For equilibrium temperatures above this, the planet is located relatively close to its host star, implying a large host star angular size that pushes the refractive boundary to pressures deeper than about 1-3 bar. Note that, for the case in Figure 6 with an atmospheric temperature of $500 \mathrm{~K}$, the planets are located at orbital distances of $0.05-0.3 \mathrm{au}$ from the hosts with effective temperatures of $3500-6000 \mathrm{~K}$, and have orbital periods of $0.01-0.2$ year.

\section{Conclusions}

Refraction and aerosol forward-scattering are complicated physical processes and accordingly are difficult to efficiently incorporate into standard transit spectrum tools. We have derived analytic expressions that can be used to easily account for scattering and a refractive boundary in transit observations and models. For scattering, we derive an effective slant optical depth that includes a correction for light that is contained in the forwardscattering peak of a aerosol-scattering phase function, and we demonstrate the accuracy of this correction by comparing to 
Table 2

Refractive Boundary Pressures for Isothermal Jupiters

\begin{tabular}{|c|c|c|c|c|c|c|c|c|c|c|}
\hline \multirow[b]{2}{*}{$\begin{array}{l}\text { Host Star } \\
\text { Spectral Type }\end{array}$} & \multirow[b]{2}{*}{$\begin{array}{c}T_{\mathrm{s}} \\
(\mathrm{K})\end{array}$} & \multicolumn{3}{|c|}{$300 \mathrm{~K}$} & \multicolumn{3}{|c|}{$600 \mathrm{~K}$} & \multicolumn{3}{|c|}{$1200 \mathrm{~K}$} \\
\hline & & $\begin{array}{l}\mathrm{B} 16^{\mathrm{a}} \\
\text { (bar) }\end{array}$ & $\begin{array}{c}\text { Equation (14) } \\
\text { (bar) }\end{array}$ & $\begin{array}{l}\text { frac. } \\
\text { error }\end{array}$ & $\begin{array}{l}\text { B16 } \\
\text { (bar) }\end{array}$ & $\begin{array}{l}\text { Equation (14) } \\
\text { (bar) }\end{array}$ & $\begin{array}{l}\text { frac. } \\
\text { error }\end{array}$ & $\begin{array}{l}\text { B16 } \\
\text { (bar) }\end{array}$ & $\begin{array}{l}\text { Equation (14) } \\
\text { (bar) }\end{array}$ & $\begin{array}{l}\text { frac. } \\
\text { error }\end{array}$ \\
\hline F0 & 7300 & 0.37 & 0.39 & 0.05 & 3.97 & 4.39 & 0.11 & 38.5 & 49.7 & 0.29 \\
\hline $\mathrm{G} 2$ & 5778 & 0.60 & 0.64 & 0.07 & 6.20 & 7.23 & 0.17 & 54.3 & 81.8 & 0.51 \\
\hline K5 & 4410 & 1.02 & 1.14 & 0.11 & 9.90 & 12.9 & 0.30 & 72.3 & 145 & 1.01 \\
\hline M2 & 3400 & 1.73 & 2.06 & 0.19 & 14.7 & 23.3 & 0.58 & 83.5 & 264 & 2.16 \\
\hline
\end{tabular}

Note.

a From Bétrémieux (2017), their Table 3.

full-physics models. Exploring this correction shows that transit spectra for exoplanets with exponentially distributed hazes can be biased by up to four scale heights when forward-scattering is ignored, depending on the angular size of the host star and the scattering asymmetry parameter. We validate the utility of a common expression for the location of a refractive boundary, which we write in terms of the refractive boundary pressure for easy incorporation into existing transit spectrum models. Using the validated expression for refractive boundaries, we show that opacity from Rayleigh scattering and CIA will outweigh refraction effects for Jupiter-like worlds with equilibrium temperatures above $400-500 \mathrm{~K}$.

T.R. gratefully acknowledges support from NASA through the Sagan Fellowship Program executed by the NASA Exoplanet Science Institute. The results reported herein benefited from collaborations and/or information exchange within NASA's Nexus for Exoplanet System Science (NExSS) research coordination network sponsored by NASA's Science Mission Directorate. Certain essential tools used in this work were developed by the NASA Astrobiology Institute's Virtual Planetary Laboratory, supported by NASA under Cooperative Agreement No. NNA13AA93A. Following acceptance of this manuscript at ApJ, the authors were made aware of García Muñoz \& Cabrera (2018), who also develop intuitive models for forward scattering effects in exoplanet transits.

\section{ORCID iDs}

Tyler D. Robinson (i) https://orcid.org/0000-0002-3196-414X Jonathan J. Fortney (i) https://orcid.org/0000-0002-9843-4354 William B. Hubbard (1) https://orcid.org/0000-0003$3185-4538$

\section{References}

Abel, M., Frommhold, L., Li, X., \& Hunt, K. L. C. 2011, JPCA, 115, 6805 Barstow, J. K., Aigrain, S., Irwin, P. G. J., et al. 2012, MNRAS, 430, 1188 Baum, W. A., \& Code, A. D. 1953, AJ, 58, 108 Beichman, C., Benneke, B., Knutson, H., et al. 2014, PASP, 126, 1134 Benneke, B., \& Seager, S. 2012, ApJ, 753, 100 Bétrémieux, Y. 2017, MNRAS, 456, 4051

Bétrémieux, Y., \& Kaltenegger, L. 2013, ApJL, 772, L31

Bétrémieux, Y., \& Kaltenegger, L. 2014, ApJ, 791, 7

Bétrémieux, Y., \& Kaltenegger, L. 2015, MNRAS, 451, 1268

Bétrémieux, Y., \& Swain, M. R. 2017, MNRAS, 467, 2834

Bideau-Mehu, A., Guern, Y., Abjean, R., \& Johannin-Gilles, A. 1973, OptCo, 9,432
Brown, T. M. 2001, ApJ, 553, 1006

Budaj, J., Kocifaj, M., Salmeron, R., \& Hubeny, I. 2015, MNRAS, 454, 2

Dalba, P. A., Muirhead, P. S., Fortney, J. J., et al. 2015, ApJ, 814, 154

De Kok, R., \& Stam, D. 2012, Icar, 221, 517

DeVore, J., Rappaport, S., Sanchis-Ojeda, R., Hoffman, K., \& Rowe, J. 2016, MNRAS, 461, 2453

Fabry, C. 1929, JO, 12, 1

Fraine, J., Deming, D., Benneke, B., et al. 2014, Natur, 513, 526

García Muñoz, A., \& Cabrera, J. 2018, MNRAS, 473, 1801

García Muñoz, A., Lavvas, P., \& West, R. A. 2017, NatAs, 1, 0114

García Muñoz, A., Zapatero Osorio, M. R., Barrena, R., et al. 2012, ApJ, 755,103

Gardner, J. P., Mather, J. C., Clampin, M., et al. 2006, SSRv, 123, 485

Henyey, L. G., \& Greenstein, J. L. 1941, ApJ, 93, 70

Hill, R. J., \& Lawrence, R. S. 1986, InfPh, 26, 371

Howe, A. R., \& Burrows, A. S. 2012, ApJ, 756, 176

Hubbard, W., Fortney, J., Lunine, J., et al. 2001, ApJ, 560, 413

Hubbard, W., Hunten, D., Dieters, S., Hill, K., \& Watson, R. 1988, Natur, 336,452

Irvine, W. M. 1965, ApJ, 142, 1563

Johnson, J. A., Aller, K. M., Howard, A. W., \& Crepp, J. R. 2010, PASP, 122, 905

Knutson, H. A., Dragomir, D., Kreidberg, L., et al. 2014, ApJ, 794, 155

Kreidberg, L. 2015, PASP, 127, 1161

Kreidberg, L., Bean, J. L., Désert, J.-M., et al. 2014, ApJL, 793, L27

Křen, P. 2011, ApOpt, 50, 6484

Lecavelier Des Etangs, A., Pont, F., Vidal-Madjar, A., \& Sing, D. 2008, A\&A, 481, L83

Lee, J.-M., Irwin, P. G. J., Fletcher, L. N., Heng, K., \& Barstow, J. K. 2014, ApJ, 789, 14

Line, M. R., \& Parmentier, V. 2016, ApJ, 820, 78

Line, M. R., Zhang, X., Vasisht, G., et al. 2012, ApJ, 749, 93

MacDonald, R. J., \& Madhusudhan, N. 2017, MNRAS, 469, 1979

Mandel, K., \& Agol, E. 2002, ApJL, 580, L171

Mansfield, C. R., \& Peck, E. R. 1969, JOSA, 59, 199

Misra, A., Meadows, V., \& Crisp, D. 2014, ApJ, 792, 61

Misra, A. K., \& Meadows, V. S. 2014, ApJL, 795, L14

Morley, C. V., Knutson, H., Line, M., et al. 2017, AJ, 153, 86

Muñoz, A. G., Osorio, M. Z., Barrena, R., et al. 2012, ApJ, 755, 103

Peck, E. R., \& Huang, S. 1977, JOSA, 67, 1550

Peck, E. R., \& Nath Khanna, B. 1966, JOSA, 56, 1059

Petty, G. 2006, A First Course in Atmospheric Radiation (Madison, WI: Sundog Publishing)

Polyanskiy, M. N. 2016, Refractive index database, http://refractiveindex.info Robinson, T. D. 2017, ApJ, 836, 236

Robinson, T. D., Maltagliati, L., Marley, M. S., \& Fortney, J. J. 2014, PNAS, 111, 9042

Schwieterman, E. W., Robinson, T. D., Meadows, V. S., Misra, A., \& Domagal-Goldman, S. 2015, ApJ, 810, 57

Seager, S., \& Sasselov, D. 2000, ApJ, 537, 916

Sidis, O., \& Sari, R. 2010, ApJ, 720, 904

Sing, D. K., Fortney, J. J., Nikolov, N., et al. 2016, Natur, 529, 59

Thomas, G., \& Stamnes, K. 1999, Radiative Transfer in the Atmosphere and Ocean, Cambridge Atmospheric and Space Science Series (Cambridge: Cambridge Univ. Press)

Waldmann, I. P., Tinetti, G., Rocchetto, M., et al. 2015, ApJ, 802, 107 